\title{
Application du procédé séchage-friture aux amandes de karité : influence sur les indices chimiques de qualité et les propriétés de fusion du beurre
}

Hilaire Macaire WOMENI $^{1}$ Robert NDJOUENKEU ${ }^{2}$

César KAPSEU ${ }^{2}$

Michel PARMENTIER ${ }^{3}$

Jacques FANNI $^{3}$

${ }^{1}$ Département de biochimie, Faculté des sciences, Université de Dschang, B.P. 67 Dschang, Cameroun, Fax : (237) 3451202

<womeni@yahoo.fr>

${ }^{2}$ Ecole nationale supérieure des sciences agroIndustrielles (ENSAI), Université de Ngaoundéré, B.P. 455 Ngaoundéré, Cameroun

${ }^{3}$ Laboratoire de Science et Génie Alimentaires, ENSAIA - INPL, 2, avenue de la forêt de Haye, 54500, Vandœuvre-les-Nancy cedex, France

\begin{abstract}
The effect of some processing parameters (thickness of kernels, kernels to butter mass ratio, temperature and duration of frying) on the acid and peroxide values and the melting properties of shea butter was studied during deep fat frying of shea kernels (Butyrospermum parkii). The results showed that the frying of kernels results in low acidic butters fulfilling commercial criteria of acidity. The increase of the frying temperature increased the peroxide value. The thermograms of butters from fried kernels dispayed a peak intermediate to both typical peaks of shea butter. The melting enthalpy of this peak increased with increasing the temperature and the duration of frying. The best frying conditions to preserve the quality of shea butter are: kernel thickness 2-6 mm; frying temperature 140-150 ${ }^{\circ} \mathrm{C}$, kernel to butter mass ratio 0,04-0,06 and duration of frying less than 10 minutes.
\end{abstract}

Key words: Butyrospermum parkii, shea butter, deep fat frying dehydration, quality

\section{Introduction}

La friture est un procédé de cuisson très utilisé pour l'élaboration de produits de type frites, beignets ou chips. Au cours de ce procédé, le produit traité subit d'abord une déshydratation due à la chaleur dégagée par l'opération. Dès lors, la friture a pu être utilisée comme un procédé de séchage pour des produits et des applications pour lesquels le séchage à l'air s'avère mal adapté (produit gras) ou pour lesquels on souhaite mener le produit à une teneur en eau très basse (moins de $4 \%$ base humide) [1]. Cette forme d'utilisation du procédé de friture reste néanmoins relativement récente. Elle s'applique par exemple au séchage des déchets d'équarrissage, et des résultats prometteurs ont été obtenus avec la noix de coco et la pulpe d'avocat [2-4]. À cet égard, la friture constitue une solution alternative intéressante pour le séchage, en vue de l'extraction d'huile, des produits à la fois riches en eau et en matières grasses [4].

Le séchage par friture avant extraction de l'huile réduit rapidement la teneur en eau et limite les risques de pollution et les réactions indésirables [4,5]. De plus, les techniques traditionnelles de séchage solaire dégradent la qualité du produit de par l'irrégularité du rayonnement solaire et la lenteur de la déshydratation. En effet, le séchage des amandes de karité au soleil ou dans un séchoir électrique à une température comprise entre 30 à $70^{\circ} \mathrm{C}$, requiert 30 à 100 heures pour ramener la teneur en eau des amandes à 10-15\%, teneur en eau optimale pour l'extraction du beurre $[6,7]$. Par séchage-friture, on atteint cette humidité nominale après environ 6 minutes [8].

Toutefois, le séchage-friture ne pourra constituer une technique alternative à la déshydratation des amandes de karité en vue de l'extraction du beurre, que s'il permet d'obtenir un beurre remplissant la qualité marchande, c'est-à-dire une acidité maximale de $9 \%$ pour le beurre à usage alimentaire [9], ou $0,3 \%$ d'acidité et 1 meq $d^{\prime} \mathrm{O}_{2} / \mathrm{kg}$ d'indice de peroxyde pour le beurre destiné à la cosmétique [10].

L'évaluation de la qualité des beurres sera complétée par l'analyse enthalpique différentielle qui est une méthode non chimique de caractérisation des propriétés de fusion de la matière grasse. Cette méthode d'investigation a l'avantage de ne nécessiter aucun produit chimique toxique, et les informations tirées du thermogramme qui en est issu présentent une excellente corrélation avec les mesures analytiques standard [11, 12].

Le présent travail a donc pour objectif d'étudier l'influence des conditions de friture des amandes de karité, d'une part sur les indices d'acide et de peroxyde, d'autre part sur les caractéristiques de fusion du beurre, dans le but final de déterminer les conditions de séchage optimal qui conduisent à l'extraction d'un beurre de qualité marchande. 


\section{Matériel et méthodes}

Les fruits de karité utilisés ont été achetés sur le marché de Ngaoundéré (Cameroun). Ils ont été ramassés à la veille du jour d'achat, sous les arbres, dans la localité de Dang, village situé dans la périphérie de la ville de Ngaoundéré. Les caractéristiques des fruits, des amandes, du beurre de karité du bain ainsi que les conditions de friture ont été précisées dans un précédent article [8]. Les amandes utilisées contiennent initialement $53,92 \pm 6,13 \%$ d'eau, $45,44 \pm 0,31 \%$ (base matière sèche) de matière grasse comprenant $36,50 \%$ d'acides gras saturés, $53,15 \%$ d'acides gras mono-insaturés et $9,58 \%$ d'acides gras polyinsaturés. Ces amandes ont été frites dans un bain de beurre de karité ayant 7,56 $\pm 1,68 \%$ d'indice d'acide et 5,48 $\pm 0,16 \mathrm{meq} / \mathrm{kg}$ d'indice de peroxyde.

L'étude de l'influence des conditions de friture sur la qualité des beurres extraits des amandes frites a été réalisée selon un plan d'expériences composite centré à quatre facteurs: l'épaisseur des amandes (2 à $18 \mathrm{~mm})$, le rapport massique amandes/huile $(0,03$ à 0,07$)$, la température du bain $\left(120\right.$ à $\left.180^{\circ} \mathrm{C}\right)$ et le temps de friture $(0,4$ à $11,6 \mathrm{~min})$. Le détail des essais a été précisé dans le même article [8].

Ce plan permet d'exprimer une variable de réponse $Y$ selon l'équation suivante :

$$
\begin{aligned}
& \mathrm{Y}=\mathrm{I}+\mathrm{ax} \mathrm{x}_{1}+\mathrm{bx} \mathrm{x}_{2}+\mathrm{cx_{3 }}+\mathrm{dx_{4 }}+\mathrm{ex_{1 }}{ }^{2}+\mathrm{fx} \mathrm{x}_{1} \mathrm{x}_{2}+\mathrm{gx} \mathrm{x}_{1} \mathrm{x}_{3}+\mathrm{hx_{1 }} \mathrm{x}_{4}+\mathrm{ix} \mathrm{x}_{2}^{2} \\
& +\mathrm{j} \mathrm{x}_{2} \mathrm{x}_{3}+\mathrm{kx} \mathrm{x}_{2} \mathrm{x}_{4}+\mathrm{l} \mathrm{x}_{3}^{2}+\mathrm{mx_{3 }} \mathrm{x}_{4}+\mathrm{nx_{4 }} \mathrm{C}_{4}^{2}
\end{aligned}
$$

Les amandes frites et refroidies à température ambiante sont ensuite broyées et les beurres extraits à l'hexane selon la méthode Soxhlet. Les indices chimiques de qualité (indice d'acide et de peroxyde) des différents échantillons de beurre obtenu ont été déterminés selon les méthodes normalisées Afnor [13].

Les analyses thermiques différentielles ont été réalisées avec un calorimètre différentiel Perkin-Elmer, modèle Pyris I (Perkin-Elmer Corp., Norwalk, USA), commandé par le logiciel Pyris I. Le calibrage a été effectué avec I'indium $(\Delta \mathrm{H}=28,43 \mathrm{~J} / \mathrm{g})$. Une masse d'échantillon d'environ $10 \mathrm{mg}$ est refroidie de la température ambiante à $-20^{\circ} \mathrm{C}$, maintenue pendant 10 minutes puis chauffée jusqu'à $60^{\circ} \mathrm{C}$ à la vitesse de $5^{\circ} \mathrm{C} / \mathrm{min}$. Les enthalpies de fusion $(\Delta \mathrm{H})$ ont été calculées par intégration de l'aire totale du pic de fusion.

Le logiciel Statgraphics Plus 5.0 a permis de construire le plan d'expériences et $d$ 'analyser la variance des coefficients de régression des équations d'expression de l'acidité, de l'indice de peroxyde et de l'enthalpie de fusion.

\section{Résultats et discussion}

\section{Indices chimiques de qualité de beurres extraits par friture}

L'acidité des beurres, exprimée en pourcentage d'acide oléique varie de 0 à $13 \%$ dans les cas extrêmes (figure $1 A, B$ et $C$ ). Les critères d'acidité des grandes firmes acheteuses de beurres de karité sont : une acidité de base de $6 \%$ avec une bonification réciproque de 1 pour 1 et une acidité maximale à l'embarquement de $9 \%$. Ceci signifie que le prix d'achat est fixé à partir de $6 \%$ de degré d'acidité. À chaque baisse de $1 \%$ d'acidité en dessous de 6 , le vendeur obtient une bonification de $1 \%$ sur le prix d'achat de base. II perd $1 \%$ sur ce prix pour chaque degré d'acidité au-dessus de 6 et ce, jusqu'à $9 \%$ d'acidité, taux d'acidité au-dessus duquel le beurre n'est plus accepté [9]. L'industrie cosmétique, qui pratique des prix jusqu'à 7 fois celui de l'industrie alimentaire [14], est plus exigeante en termes de qualité : le taux souhaité est $0,3 \%$ d'indice d'acide [10]. Par rapport à ces critères de marché, le beurre de karité extrait de la plupart des échantillons présente une acidité inférieure aux $9 \%$ de la borne supérieure de l'industrie alimentaire, et reste dans la
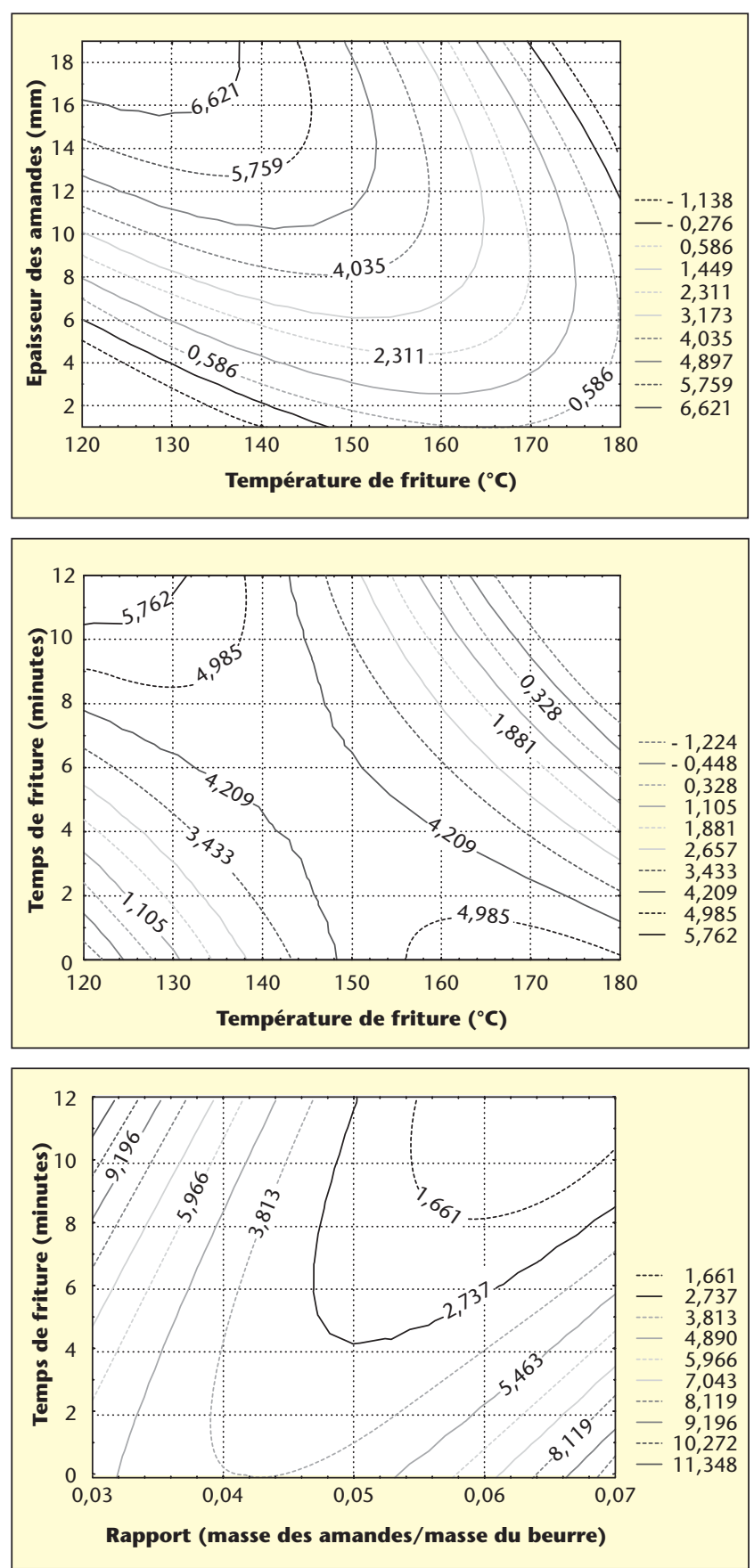

Figure 1. Courbes isoréponses montrant la variation de l'acidité du beurre (\% acide oléique) en fonction (A) de la température de friture et de l'épaisseur des amandes, (B) de la température et du temps de friture, $(C)$ du temps de friture et du rapport massique amandes/beurre.

majorité des cas supérieure à $0,3 \%$ ce qui le disqualifie pour une application en cosmétique $[9,10]$.

Les coefficients de l'équation de régression, la probabilité et les coefficients de détermination d'équation générée par les résultats de l'acidité des beurres sont donnés dans le tableau 1. On peut remarquer que le modèle généré explique $62,19 \%$ de variation de l'acidité des beurres. $38 \%$ des variations dépendent donc en partie des variables qui n'ont pas été prises en compte ici. L'analyse des coefficients du modèle mathématique généré par les variables de commande étudiées et leurs interactions 
Tableau 1. Coefficients de l'équation de régression $(C R)$, probabilité $(P)$ et coefficient de détermination de l'analyse des variances des modèles d'expression de l'acidité, de l'indice de peroxyde et de l'enthalpie de fusion des composés des pics intermédiaires des beurres extraits des amandes déshydratées par friture selon le plan d'expérience composite centré.

\begin{tabular}{|c|c|c|c|c|c|c|}
\hline \multirow[t]{2}{*}{ Sources } & \multicolumn{2}{|c|}{ Acidité } & \multicolumn{2}{|c|}{ Indice de peroxyde } & \multicolumn{2}{|c|}{$\begin{array}{l}\text { Enthalpie de fusion } \\
\text { des pics intermédiaires }\end{array}$} \\
\hline & CR & $\mathbf{P}$ & CR & $\mathbf{P}$ & CR & $\mathbf{P}$ \\
\hline $\mathrm{x}_{1}$ : Épaisseur des amandes & 2,5735 & $0,001^{\mathrm{a}}$ & 11,9113 & 0,258 & $-1,0874$ & 0,147 \\
\hline$x_{2}:$ Température de friture & 0,8246 & $0,021^{\mathrm{a}}$ & 1,9086 & $0,000^{\mathrm{a}}$ & $-2,1544$ & $0,000^{\mathrm{a}}$ \\
\hline$x_{3}:$ Rapport massique & $-1030,01$ & $0,023^{\mathrm{a}}$ & 4219,02 & 0,477 & $-3061,0$ & $0,001^{\mathrm{a}}$ \\
\hline $\mathrm{x}_{4}:$ Temps de friture & 5,5235 & 0,250 & $-10,6065$ & $0,000^{\mathrm{a}}$ & $-10,711$ & $0,000^{\mathrm{a}}$ \\
\hline$x_{1}^{2}$ & $-0,0131$ & 0,563 & $-0,2456$ & $0,000^{\mathrm{a}}$ & 0,0155 & 0,699 \\
\hline$x_{1} x_{2}$ & $-0,0157$ & $0,017^{\mathrm{a}}$ & $-0,0547$ & $0,003^{\mathrm{a}}$ & $-0,0002$ & 0,984 \\
\hline$x_{1} x_{3}$ & 3,8826 & 0,683 & 0,8791 & 0,973 & 9,0437 & 0,589 \\
\hline$x_{1} x_{4}$ & 0,0196 & 0,563 & 0,1538 & 0,103 & 0,0265 & 0,658 \\
\hline$x_{2}^{2}$ & $-0,0023$ & 0,160 & $-0,0028$ & 0,525 & 0,0059 & $0,042^{\mathrm{a}}$ \\
\hline$x_{2} x_{3}$ & 2,7380 & 0,283 & $-13,3601$ & 0,059 & 5,6175 & 0,213 \\
\hline$x_{2} x_{4}$ & $-0,0272$ & $0,004^{\mathrm{a}}$ & 0,0821 & $0,002^{\mathrm{a}}$ & 0,0583 & $0,001^{\mathrm{a}}$ \\
\hline$x_{3}^{2}$ & 7240,31 & 0,052 & $-21319,0$ & $0,037^{a}$ & 21828,5 & $0,001^{a}$ \\
\hline$x_{3} x_{4}$ & $-36,2095$ & $0,011^{\mathrm{a}}$ & $-25,3677$ & 0,494 & 24,125 & 0,316 \\
\hline$x_{4}^{2}$ & 0,0031 & 0,947 & $-0,0573$ & 0,651 & 0,1079 & 0,192 \\
\hline Constante & $-57,4175$ & & $-283,733$ & & 251,199 & \\
\hline Coefficient de détermination (\%) & \multicolumn{2}{|c|}{62,189} & \multicolumn{2}{|c|}{74,252} & \multicolumn{2}{|c|}{75,548} \\
\hline
\end{tabular}

a Valeurs de $p<0,05$, indiquant qu'il y a effet significatif de 0 à $95 \%$ niveau de confidentialité.

montre que le terme de premier ordre de l'épaisseur des amandes $\left(x_{1}\right)$ et le terme d'interaction de la température de consigne de friture et le temps de friture $\left(x_{2} x_{4}\right)$ ont des effets significatifs très importants $(P<0,01)$ sur l'acidité des beurres. Les termes de premier ordre de la température de friture $\left(\mathrm{x}_{2}\right)$, du rapport massique amandes/beurre $\left(\mathrm{x}_{3}\right)$, les termes d'interactions de l'épaisseur des amandes et de la température de friture $\left(x_{1} x_{2}\right)$, du rapport massique amandes/beurre et du temps de friture $\left(x_{3} x_{4}\right)$ ont également des effets significatifs $(P<0,05)$ sur l'acidité des beurres.

L'augmentation de l'épaisseur des tranches d'amandes entraîne une augmentation de l'acidité du beurre. Lorsque la friture s'effectue entre 130 et $140{ }^{\circ} \mathrm{C}$, les amandes de $16 \mathrm{~mm}$ d'épaisseur donnent un beurre d'acidité de l'ordre de $6 \%$, alors que celles de $4 \mathrm{~mm}$ d'épaisseur donnent un beurre d'environ $0,5 \%$ d'acidité (figure $1 A$ ). II ressort de la figure $1 B$ qu'à moins de $150^{\circ} \mathrm{C}$, le prolongement du temps de friture des amandes acidifie le beurre extrait. Au bout de 4 minutes de friture à $130^{\circ} \mathrm{C}$, on obtient des beurres avec 2 à $3 \%$ d'acidité. En restant à la même température et en prolongeant la friture jusqu'à 10 minutes, on obtient des beurres avec $5 \%$ d'acidité. Mais lorsque la température de friture est de l'ordre de 160 à $180^{\circ} \mathrm{C}$, l'acidité est inférieure à $3 \%$ pour des durées de friture entre 6 et 12 minutes. La figure $1 \mathrm{C}$ montre que les acidités de 1 à $3 \%$ sont obtenues avec les amandes frites dans un rapport massique de 0,045 à 0,070 pendant plus de 4 minutes. Lorsqu e ce rapport est inférieur à 0,045 et combiné à un temps de friture supérieur à 4 minutes ou alors s'il est entre 0,06-0,07 et associé à un temps inférieur à 4 minutes, l'acidité augmente et peut atteindre $10 \%$.

L'immersion brutale des amandes dans un bain porté à plus de $100^{\circ} \mathrm{C}$, inhiberait l'activité de leurs enzymes lipolytiques naturelles. Cependant, l'augmentation de l'épaisseur des amandes ralentit la vitesse de transfert de chaleur et empêche d'atteindre rapidement des températures d'inactivation des enzymes au cœur du produit notamment lorsque la friture $s^{\prime}$ effectue entre 120 et $140^{\circ} \mathrm{C}$. Dans ces conditions, il aurait activation transitoire des enzymes endogènes qui catalysent l'hydrolyse des triglycérides pendant les traitements post-friture (refroidissement, broyage et extraction de la matière grasse). Le même phénomène serait observé lorsque le rapport massique amandes/huile est supérieur à 0,06 et le temps de friture inférieur à 4 minutes. Lorsque le temps de friture s'allonge, il s'en suit une augmentation de la quantité d'huile provenant du bain qui est absorbée par l'amande. Ceci a été rapporté par Womeni et al. [8] avec des amandes de karité et par Sosa-Morales et al. [15] avec la viande de porc. L'acidité du bain de friture étant plus élevée que celle des amandes, son absorption entraînerait une augmentation de l'acidité de la matière grasse des amandes. En effet, il est connu qu'en friture profonde longue, la composition des lipides du produit tend vers celle du bain de friture $[16,17]$.

L'acidification du beurre est minimale lors de la friture de tranches d'amandes d'épaisseur inférieure à $8 \mathrm{~mm}$, à une température comprise entre 140 et $180^{\circ} \mathrm{C}$, pendant 4 à 6 minutes et dans le rapport massique 0,045-0,070.

$\mathrm{L}^{\prime}$ indice de peroxyde des beurres, exprimé en meq $\mathrm{d}^{\prime} \mathrm{O}_{2} / \mathrm{kg}$ de beurre, varie de 0 à $37 \mathrm{meq} / \mathrm{kg}$ (figure $2 A$ et B). Dans l'ensemble, les valeurs obtenues sont supérieures à $1 \mathrm{meq} / \mathrm{kg}$ qui est la valeur maximale du beurre de karité souhaitée en cosmétique [10]. En alimentation, la borne supérieure d'indice de peroxyde préconisée pour les huiles de consommation est plus variée : elle est de l'ordre de $1 \mathrm{meq} / \mathrm{kg}$ pour les huiles alimentaires courantes, mais peut aller jusqu'à $10 \mathrm{meq} / \mathrm{kg}[18,19]$. Pour les beurres de karité dont les conditions de maturité et de récolte sont variables, certains auteurs proposent jusqu'à $20 \mathrm{meq} / \mathrm{kg}$ [20], $70 \mathrm{meq} / \mathrm{kg}$ [21], voire $125 \mathrm{meq} / \mathrm{kg}$ [22]. De telles matières grasses apparaissent toutefois déjà fortement dégradées par oxydation et sont impropres à la consommation humaine.

L'analyse de la variance des coefficients générés par le modèle mathématique (tableau 1) montre que $74,25 \%$ des variations de l'indice de peroxyde s'expliquent par les variations de l'épaisseur des tranches d'amandes, de la température de consigne de friture, du rapport massique amandes/beurre du bain et du temps de friture. Les effets les plus significatifs $(P<0,01)$ sont ceux du terme de second ordre de l'épaisseur des amandes $\left(\mathrm{x}_{1}{ }^{2}\right)$, des termes de premier ordre de la température de friture $\left(x_{2}\right)$ et du temps de friture $\left(x_{4}\right)$. D'autres effets significatifs $(P<0,05)$ sont ceux du terme de second ordre du rapport massique amandes/beurre $\left(x_{3}{ }^{2}\right)$ et des termes d'interactions épaisseur des amandes et température de friture $\left(x_{1} x_{2}\right)$, température de friture et temps de friture $\left(x_{2} x_{4}\right)$. 

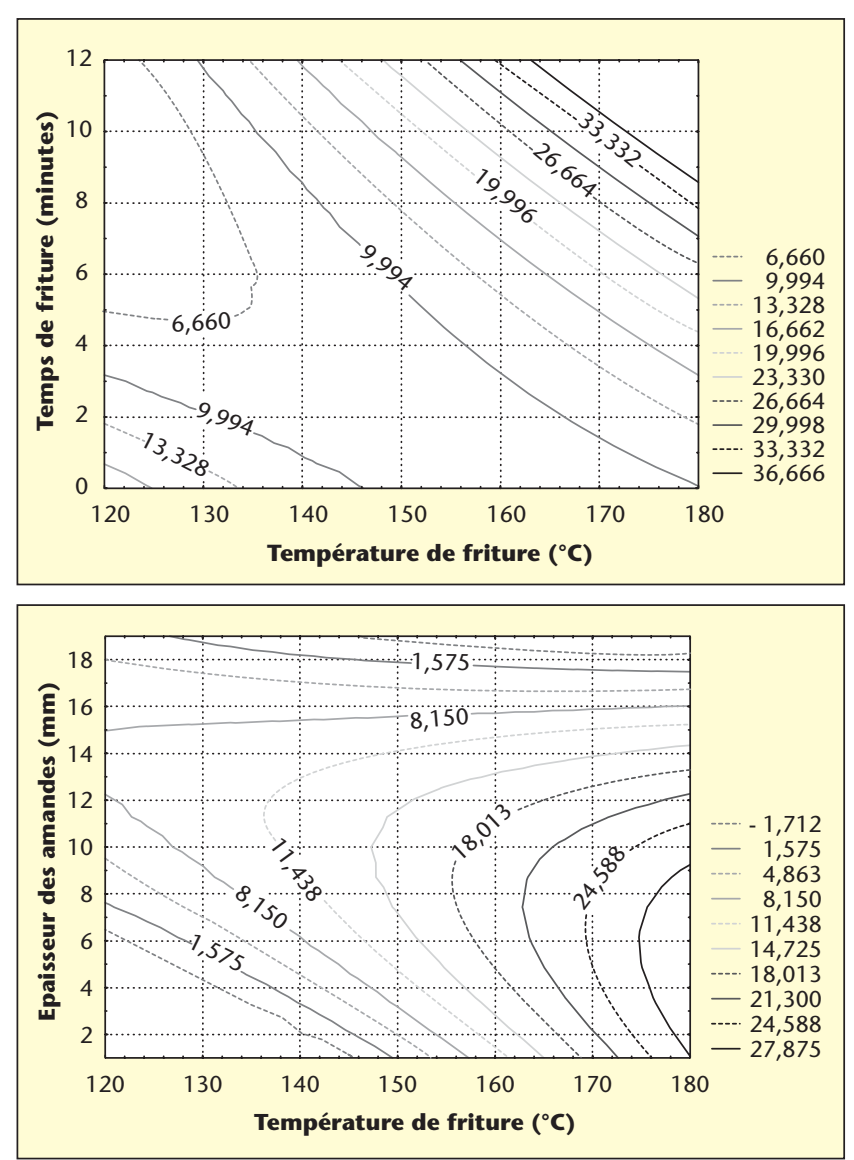

Figure 2. Courbes isoréponses montrant la variation de l'indice de peroxyde du beurre (meq d' $\mathrm{O}_{2} / \mathrm{kg}$ ) en fonction ( $A$ ) de la température de friture et du temps de friture, (B) de la température de friture et de l'épaisseur des amandes.

Les courbes isoréponses tirées de ces résultats (figure $2 A$ et $B$ ) montrent que l'augmentation de la température et du temps de friture s'accompagne d'une augmentation de l'indice de peroxyde (figure 2A). L'indice de peroxyde est élevé avec les amandes de 6 à $14 \mathrm{~mm}$ d'épaisseur et baisse de part et d'autre de ces valeurs (figure $2 B$ ). Cette accélération de la phase primaire de l'oxydation consécutive à l'élévation de température avait déjà été notée par Gere [23]. En outre, l'élévation de la température favorise la formation de composés potentiellement toxiques tels que les monomères cycliques. En conséquence, les températures de friture très élevées doivent être évitées.

En tenant compte des conditions exprimées ci-dessus concernant l'acidification, les paramètres de conduite du procédé de friture qui minimisent à la fois l'hydrolyse et l'oxydation de la matière grasse des amandes de karité sont les suivantes: température de friture de 140 à $150^{\circ} \mathrm{C}$, épaisseur des amandes inférieure à $4 \mathrm{~mm}$, rapport massique amandes/beurre de 0,045 à 0,070 et temps de friture de 2 à 6 minutes.

\section{Propriétés de fusion des beurres extraits par friture}

Les thermogrammes des beurres extraits des amandes frites présentent trois pics. Deux pics dits «classiques» observés dans tous les thermogrammes des beurres de karité : le premier, caractéristique des bas points de fusion entre -10 et $+10{ }^{\circ} \mathrm{C}$ et le second entre 25 et $45^{\circ} \mathrm{C}$, représentant les haut points de fusion. Un troisième pic apparaît à une température intermédiaire entre 10 et $25^{\circ} \mathrm{C}$ (figure 3). L'analyse statistique des enthalpies des composés des pics intermédiaires a permis de générer une

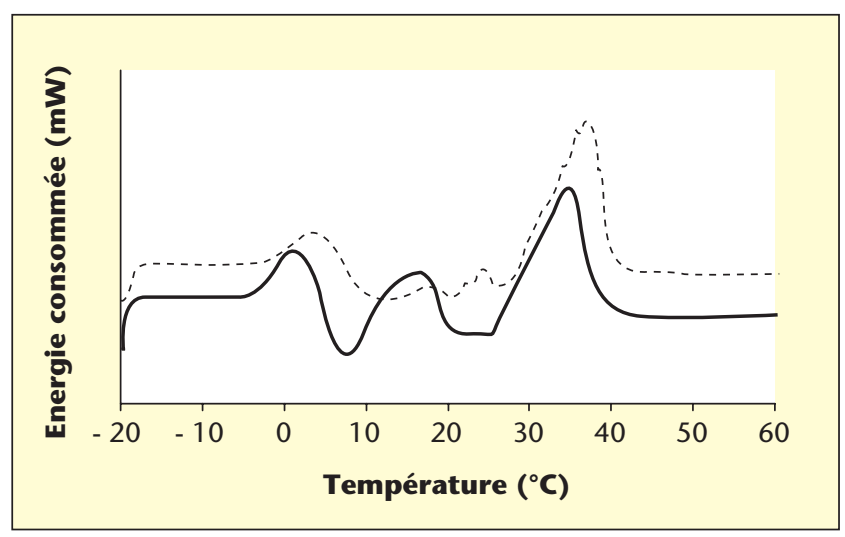

Figure 3. Thermogrammes de deux échantillons de beurre extrait des amandes frites. Pointillés: Amandes de $6 \mathrm{~mm}$ d'épaisseur frites à $135^{\circ} \mathrm{C}$ dans le rapport massique amandes/beurre de 0,04 pendant 8,8 minutes. Trait plein: Amandes de $14 \mathrm{~mm}$ d'épaisseur frites à $165^{\circ} \mathrm{C}$ dans le rapport massique amandes/beurre de 0,06 pendant 8,8 minutes.

équation exprimant cette enthalpie en fonction de l'épaisseur des amandes, de la température de friture, du rapport massique et du temps de friture. Les coefficients de cette équation sont donnés dans le tableau 1. La variation de l'enthalpie du pic intermédiaire dépend à $76 \%$ de la température du bain de friture, du rapport massique amandes/beurre et de la durée de friture. Les effets significatifs à $\mathrm{P}<0,01$ sont ceux du terme de second ordre du rapport massique amandes/beurre $\left(\mathrm{x}_{3}{ }^{2}\right)$, des termes du premier ordre de la température de friture $\left(x_{2}\right)$, du rapport massique amandes/beurre $\left(x_{3}\right)$, du temps de friture $\left(x_{4}\right)$ et du terme $d^{\prime}$ interactions température et temps de friture $\left(x_{2} x_{4}\right)$. L'effet significatif à $P<0,05$ est celui du terme de second ordre de la température $\left(\mathrm{x}_{2}{ }^{2}\right)$. L'effet de l'épaisseur des amandes n'est pas significatif. D'une manière générale, les conditions de friture qui conduisent à des enthalpies élevées de ce pic associent des températures de friture de 150 à $180^{\circ} \mathrm{C}$ à des temps de friture de 4 à 12 minutes (figure $4 A$ ) sous des rapports massiques de 0,05 à 0,07 (figure 4B).

Dans les paragraphes précédents, il a été noté que les températures de 150 à $180^{\circ} \mathrm{C}$ associés à un allongement de la durée de friture entraînent l'augmentation des hydroperoxydes, alors que l'augmentation du rapport massique est favorable à la production d'acides gras libres et de glycérides partiels. D'une part, la présence des hydroperoxydes dans les huiles perturbe leur arrangement polymorphique et déplace les températures de fusion vers les basses températures $[12,24]$. Par ailleurs, la présence des acylglycérols partiels tend à abaisser la température de fusion des mélanges de glycérides [24]. Compte tenu de ces éléments, le pic intermédiaire peut être attribué aux acides gras libres et aux mono- et diglycérides présents en quantité plus importante lorsque la friture des amandes humides est menée entre 150 à $180^{\circ} \mathrm{C}$ et/ou pendant 4 à 12 minutes. Ces conditions devraient être évitées pour minimiser l'enthalpie du pic intermédiaire signe d'apparition des produits de dégradation par hydrolyse de la matière grasse des amandes.

\section{Conclusion}

L'étude de la qualité des beurres issus des amandes frites a montré qu'en général ces beurres ont des teneurs acceptables en acides gras libres. L'augmentation de l'indice de peroxyde est notée avec les beurres des amandes frites aux fortes températures. Les thermogrammes des beurres présentent un pic intermédiaire dont l'enthalpie de fusion croît avec l'augmentation de la température et du temps de friture. Ceci est lié à 

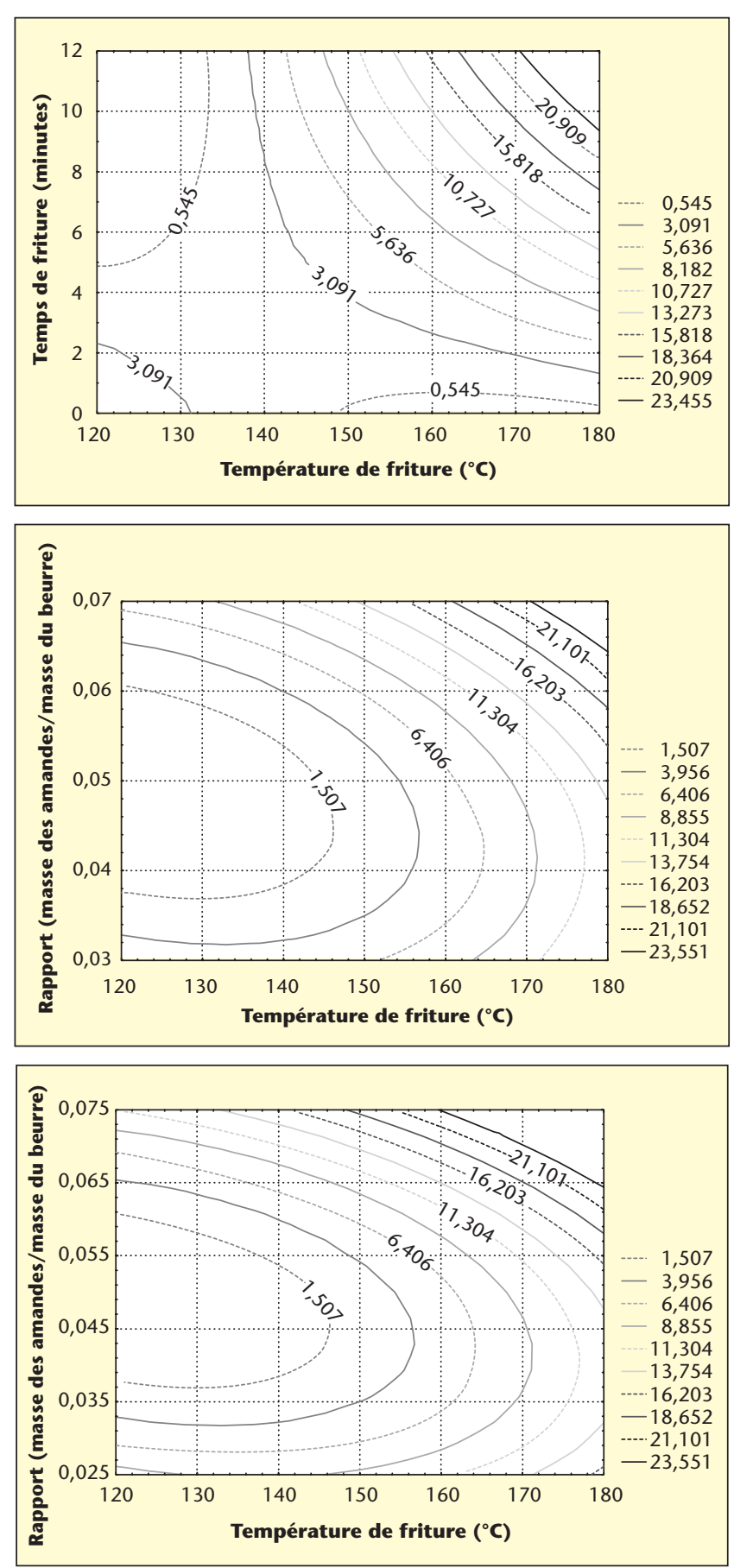

Figure 4. Courbes isoréponses montrant la variation de l'enthalpie de fusion $(\mathrm{J} / \mathrm{g}) \mathrm{du}$ pic intermédiaire en fonction (A) de la température et du temps de friture (B) de la température de friture et du rapport massique amandes/beurre.

l'apparition des acides gras libres et des glycérides partiels, produits de dégradation de la matière grasse. Compte tenu des modifications induites par la friture des amandes sur la qualité marchande du beurre, les meilleures conditions de friture, optimisées à l'aide du plan d'expériences mis en œuvre sont : épaisseur des amandes entre 2 et $6 \mathrm{~mm}$; température de friture entre 140 et $150^{\circ} \mathrm{C}$, rapport massique de 0,04 à 0,06 et temps de friture inférieur à 10 minutes.
Remerciements. Ce travail a été financé par la Fondation Internationale pour la science (IFS, Stockholm, Suède) et I'Université des Nations Unies (UNU, Tokyo, Japon) à travers I'allocation de recherche $N^{\circ}$ E/3409-1 accordée au premier auteur. Certaines analyses ont été effectuées au Laboratoire de science et génie alimentaires (LSGA) de I'Institut national polytechnique de Lorraine (INPL, Nancy, France); les missions ont été supportées par I'Institut de recherche pour le développement (IRD, Paris, France).

\section{RÉFÉRENCES}

1. VITRAC O, LISSE I, TRYSTRAM G, RAOULT-WACK AL. In : Séchage par friture, transformations physiques et biochimiques. $16^{e}$ journées de I'AFSIA, Séchage avec réaction, 28-29 avril 1998. Association Française de Séchage dans I'Industrie et l'Agriculture, $1998:$ : 27-35.

2. MERION E. Application du procédé séchage-friture à l'avocat en vue de l'extraction d'huile à usages cosmétiques, Stage de fin d'études, CIRAD/SAR n45/95. Université de Montpellier, 1995.

3. HOUNHOUIGAN I, ROUZIERE A, NOËL JM, BRICAS N, MAROUZE C, RAOULT-WACK AL. Relance de la filière de production d'huile de coco par la technique de séchage friture. Récents progrès en Génie des procédés 1997 ; 11(59) : 121-30.

4. RAOULT-WACK AL, ROUZIÈRE A, REYNES M, NOËL JM. An original aplication of deep-fat frying dehydratation of water-rich oily materials prior to oil extraction. In : Howitt R, ed. Seventh International Congress on Engineering and Food, ICEF97. Sheffield, UK : Scheffield Academic Press, 1997 : 49-52.

5. DREW PJ, BREAG GR, MARDER RC. Coprah production using the coconut shell carbonization with wasteheat recovery technology. Tropical Science $1993 ; 33: 246-67$

6. WOMENI HM, KAMGA R, TCHIEGANG C, KAPSEU C. Extraction du beurre de karité : influence du séchage des amandes et de la technique d'extraction. La Rivista Italiana Delle Sostanze Grasse 2002 ; LXXIX : 33-7.

7. WOMENI HM. Identification et analyse des opérations critiques de préparation des fruits, graines et amandes de karité (Butyrospermum parkii (G. Don) Kotschy): étude de leur influence sur la qualité du beurre. Thèse de Doctorat/Ph.D en technologie alimentaire, ENSAl, Université de Ngaoundéré, 2004.

8. WOMENI HM, NDJOUENKEU R, KAPSEU C, PARMENTIER M. Application du procédé séchage-friture aux amandes de karité : cinétique de séchage. $O C L$ $2004 ; 11(6)$ : 457-63.

9. KASSAMBA B. In : Synthèse des techniques connues d'extraction et de conditionnement du beurre de karité au Burkina Faso. Rapport final. Ouagadougou : Projet filière karité du CECI IRSAT, 1997 : 3-9.

10. DEFEZ G. Traitement du beurre de karité. Stéarinerie DUBOIS FILS, 1992. In : Kassamba B, ed. Synthèse des techniques connues d'extraction et de conditionnement du beurre de karité au Burkina Faso. Rapport final. Ouagadougou : Projet filière karité du CECI IRSAT, 1997 : 3-9.

11. TAN CP, CHE MAN YB. Differential scanning calorimetric analysis for monitoring the oxidation of heated oils. Food Chem $1999 ; 67$ : 177-84.

12. TAN CP, CHE MAN YB, SALAMAT I, YUSOFF MSA. Comparative studies of oxidative stability of edible oils by differential scanning calorimetry and oxidative stability index methods. Food Chem $2002 ; 76$ : 385-9.

13. AFNOR. Recueil des normes françaises. Corps gras graines oléagineuses, produits dérivés. $2^{e}$ édition. Paris : Afnor, 1981.

14. APROMA. Etude de la filière karité du Burkina Faso, Volume 1: Rapport principal. Ouagadougou : Union Européenne, Délégation de la commission européenne du Burkina Faso, 1995.

15. SOSA-MORALES ME, ORZUNA-ESPIRITU R, VELEZ-RUIZ JF. Mass, thermal and quality aspects of deep-fat frying of pork meat. J Food Engineering 2005 ; (article in press). 
16. SANCHEZ-MUNIZ FJ, VIEJO JM, MEDINA R. Deep-frying of sardines in different culinary fats. Changes in the fatty acid composition of sardines and frying fats. J Agric Food Chem $1992 ; 40$ : 2252-6.

17. ROSARIO RAMIREZ M, CAVA R. Changes in colour, lipid oxidation and fatty composition of pork loin chops as affected by the type of culinary frying fat. LWT 2005 ; 38 : 726-34.

18. CODEX ALIMENTARIUS. Programme mixte FAO/OMS sur les normes alimentaires. Rome (Italie) : FAO, 1992.

19. HAROLD E, RONALD SK, RONALD S. Oils and Fats in chemical analysis of food. Edinburgh, London : Churchill, Livingstone, 1981.
20. WOLFF JP. Analyse et dosage des lipides. In: Techniques d'analyses et de contrôle dans les industries agroalimentaires, 4, Analyse des constituants alimentaires. Paris : Lavoisier, Tec et Doc, 1991.

21. HART LF, FISHER JH. Oils and fats. In : Modern food analysis. Springer-Verlag, New york : Heidelberg Berlin Inc, 1971.

22. JACOBS MB. Chemical analysis of foods. $3^{\text {rd }}$ ed. New York : D. Van Nostrand Co., 1965.

23. GERE A. A complex Procedure for the Analysis of Used Frying Fats. Fette Seifen, Anstrichmittel $1983 ; 85: 111-7$.

24. CHE MAN Y, SWE PZ. Thermal analysis of failed-batch palm oil by differential scanning colorimetry. J Am Oil Chem Soc $1995 ; 72$ : 1529-32. 